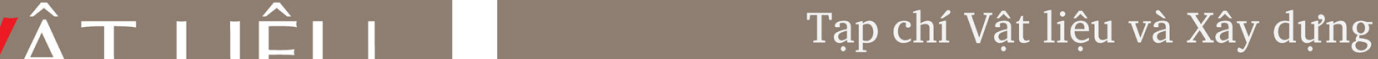 \\ ISSN 1859 - 381X \\ Website: www. jomc.vn
}

\section{Ảnh hưởng của cốt liệu được chế tạo từ tro bay thay thế cát tự nhiên tới một số tính chất của vữa xi măng}

\author{
Lưu Thị Hồng ${ }^{*}$, Đào Công Anh², Hoàng Lê Trung ${ }^{2}$, Nguyễn Hải Long ${ }^{2}$, Nobuo Inoue ${ }^{3}$ \\ ${ }^{1}$ Viện Vật liệu Xây dựng \\ ${ }^{2}$ Công ty cổ phần INA \\ ${ }^{3}$ Công ty Abe Iron work
}

TỪ KHOÁ

Cốt liệu tro bay

Vữa xi măng

Cát tự nhiên

\section{TÓM TÁ́T}

Bài báo này trình bày kết quả nghiên cứu ảnh hưởng của cốt liệu mịn được chế tạo từ tro bay thay thế cát tự nhiên tới tính chất của vữa xi măng. Vữa sử dụng cốt liệu từ tro bay có kích thước từ $4,5 \mathrm{~mm}$ đến 0,14 $\mathrm{mm}$ cho tính chất tốt hơn vữa sử dụng cốt liệu từ tro bay kích thước nhỏ hơn $2,5 \mathrm{~mm}$ và lượng hạt dưới kích thước $0,14 \mathrm{~mm}$ lớn hơn $25 \%$. Hàm lượng cốt liệu từ tro bay thay thế cát tăng thì khối lượng riêng của vữa giảm tương ứng, nhu cầu nước cho vữa tăng nguyên nhân do các hạt cốt liệu từ tro bay có độ rỗng xốp và hút nước lớn. Cường độ nén của vữa ở tuổi 28 ngày tương đương mẫu đối chứng khi sử dụng $40 \%$ hạt cốt liệu từ tro bay thay cát tự nhiên. Kết quả nghiên cứu này là bước đầu chứng minh khả năng ứng dụng sử dụng hạt cốt liệu từ tro bay thay thế cát tự nhiên để chế tạo vữa và bê tông.

\begin{abstract}
This paper presents the results of investigation on the effect of fly ash granules to replace natural sand to the properties of cement mortar. Mortar used fly ash granules size from $4.5 \mathrm{~mm}$ to $0.14 \mathrm{~mm}$ had better properties than mortar used fly ash granules size of less than $2.5 \mathrm{~mm}$ and the amount of particles below of $0.14 \mathrm{~mm}$ size was more than $25 \%$ by weight. The fly ash granules content to replace sand increased it achieve bulk density of mortar decreases, the water demand for the mortar increases due to the high porosity of fly ash granules. The mortar compressive strength at 28 days had equivalent control sample compressive strength, which used of $40 \%$ fly ash granules to replace natural sand. This research result is the first step to demonstrate the applicability used fly ash granules to replace natural sand for mortar and concrete.
\end{abstract}

\section{Giới thiệu}

Cốt liệu nhân tạo được các nước trên thế giới nghiên cứu và sử dụng phổ biến trong chế tạo vữa và bê tông. Trong đó cốt liệu nhân tạo từ tro bay thay thế cốt liệu tự nhiên cũng được các nước sử dụng và đưa vào nhóm cốt liệu nhẹ, phổ biến là tiêu chuẩn Mỹ ASTM C331và ASTM C332 quy định cốt liệu từ tro bay có kích thước hạt từ 4,75 mm tới $0 \mathrm{~mm}$; khối lượng thể tích đổ đống không lớn hơn $1120 \mathrm{~kg} / \mathrm{m}^{3}$ và một số tính chất đặc trưng khác khi ứng dụng trong các điều kiện cụ thể. Trên thế giới có nhiều phương pháp khác nhau chế tạo cốt liệu từ tro bay như: nung tro bay tới nhiệt độ kết khối sau đó nghiền sàng và tạo hạt, tạo hạt tro bay bằng chất kết dính polymer, tạo hạt tro bay bằng chất kết dính xi măng... Mỗi phương pháp tạo hạt cốt liệu từ tro bay có công nghệ khác nhau, trong nghiên cứu này hạt cốt liệu từ tro bay được chế tạo bằng chất kết dính xi măng (10 \% theo khối lượng) trên thiết bị trộn Vibro Explorer.

\section{Vật liệu và phương pháp nghiên cứu}

\subsection{Các phương pháp tiêu chuẩn được sử dung nghiên cứu gồm}

+ TCVN 6016 : 2011 - Xác định cường độ của xi măng ở điều kiện tiêu chuẩn tuổi 3 và 28 ngày;

+ TCVN 141 : 2008 - Xác định thành phần hóa học của clanhke

xi măng;

+ TCVN 8654 : 2011 - Xác định hàm lượng nước liên kết và hàm lượng sunfua trioxit tổng của thạch cao.

\subsection{Phương pháp tạo hạt từ tro bay}

Tro bay và xi măng được trộn với nước theo tỷ lệ được thể hiện trong Bảng 2.1. Hỗn hợp vật liệu được tạo hạt bằng thiết bị Vibro Explore với năng suất $250 \mathrm{~kg} / \mathrm{h}$. Thiết bị tạo hạt được thể hiện trong Hình 2.1.

Hạt cốt liệu từ tro bay đạt kích thước nhỏ hơn 4,5 mm là 90 \%, lượng nước trộn tối ưu là $25 \%$ so với tổng khối lượng. Sau khi tạo hạt, tro bay được dưỡng hộ ẩm đến thời gian 28 ngày. 


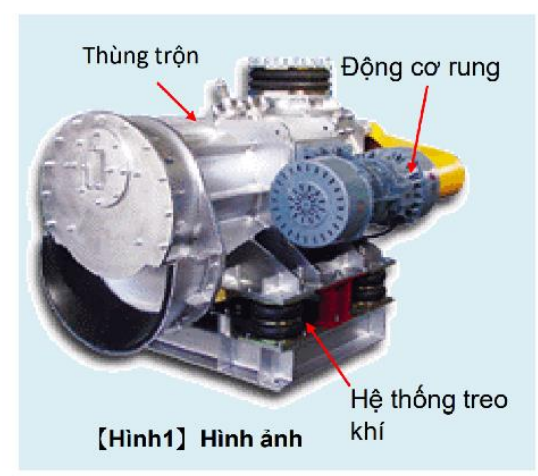

Hình 2.1.a. Thiết bị Vibro Explorer

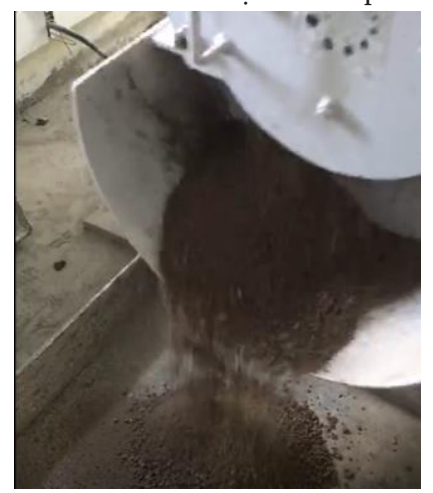

Hình 2.1.b. Tạo hạt từ tro bay

Bảng 2.1. Tỷ lệ thành phần phối trộn để tạo hạt từ tro bay.

\begin{tabular}{|l|c|c|c|}
\hline & Xi măng, \% & Tro bay, \% & Nước trộn,\% \\
\hline Hạt tro bay & 10 & 90 & 25 \\
\hline
\end{tabular}

\subsection{Vật liệu}

Các nguyên liệu sử dụng trong nghiên cứu là clanhke xi măng pooc lăng Hoàng Thạch, tro bay nhà máy nhiệt điện Thăng Long - Quảng Ninh, Thạch cao FGD Nghi Sơn.

\subsubsection{Clanhke xi măng Hoàng Thạch}

Clanhke có thành phần hóa học và thành phần khoáng được thể hiện trong Bảng 2.2, Bảng 2.3 và Hình 2.1.

Bảng 2.2. Thành phần hóa học của clanhke xi măng

\begin{tabular}{|c|c|c|c|c|c|c|c|c|c|}
\hline $\begin{array}{c}\text { Thành } \\
\text { phần }\end{array}$ & $\mathrm{SiO}_{2}$ & $\mathrm{Al}_{2} \mathrm{O}_{3}$ & $\mathrm{Fe}_{2} \mathrm{O}_{3}$ & $\mathrm{CaO}$ & $\mathrm{MgO}$ & $\mathrm{K}_{2} \mathrm{O}$ & $\mathrm{Na}_{2} \mathrm{O}$ & $\mathrm{SO}_{3}$ & $\mathrm{MKN}$ \\
\hline$\%$ & 21,89 & 5,26 & 3,56 & 65,92 & 1,23 & 0,11 & 0,012 & 0,01 & 0,56 \\
\hline
\end{tabular}

Bảng 2.3. Thành phần khoáng của clanhke xi măng.

\begin{tabular}{|c|c|c|c|c|}
\hline Thành phần & $\mathrm{C}_{3} \mathrm{~S}$ & $\mathrm{C}_{2} \mathrm{~S}$ & $\mathrm{C}_{3} \mathrm{~A}$ & $\mathrm{C}_{4} \mathrm{AF}$ \\
\hline$\%$ & 61,54 & 16,33 & 7,92 & 10,83 \\
\hline
\end{tabular}

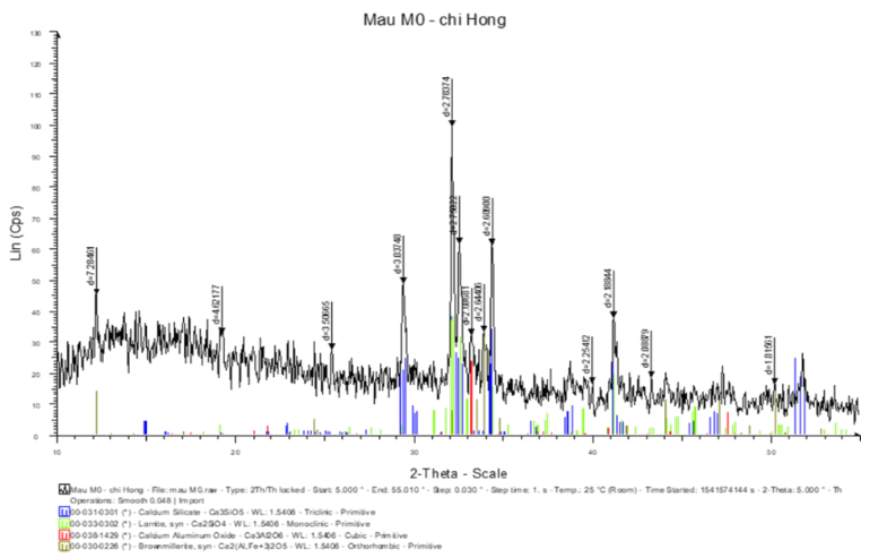

Hình 2.1. Giản đồ XRD của clanhke xi măng.

Clanhke xi măng được nghiền với $4 \%$ thạch cao để tạo thành xi măng. Tính chất cơ lý của xi măng được thể hiện trong Bảng 2.4.

Bảng 2.4. Tính chất cơ lý của xi măng.

\begin{tabular}{|c|c|c|}
\hline \multicolumn{2}{|c|}{ Tính chất } & Kết quả \\
\hline \multicolumn{2}{|l|}{ 1. Độ ẩm, \% } & 0,46 \\
\hline \multicolumn{2}{|c|}{ 2. Độ mịn còn lại trên sàng 0,09 mm , \% } & 2,15 \\
\hline \multicolumn{2}{|c|}{ 3. Độ mịn còn lại trên sàng 0,045 mm , \% } & 11,7 \\
\hline \multicolumn{2}{|l|}{ 4. Cường độ nén, MPa } & \\
\hline \multicolumn{2}{|l|}{ - Tuổi 3 ngày } & 26,3 \\
\hline \multicolumn{2}{|l|}{ - Tuổi 28 ngày } & 47,2 \\
\hline \multicolumn{2}{|l|}{ 5. Nước tiêu chuẩn, \% } & 30 \\
\hline \multirow[t]{2}{*}{ 6. Thời gian đông kết, phút } & Bắt đầu & 89 \\
\hline & Kết thúc & 170 \\
\hline
\end{tabular}

\subsubsection{Thach cao}

Thạch cao được sử dụng trong nghiên cứu là thạch cao FGD của nhà máy nhiệt điện Nghi Sơn có thành phần hóa học thể hiện trong Bảng 2.5 và các tính chất cơ lý thể hiện trong Bảng 2.6. Thạch cao FGD có màu xám, dạng bột (Hình 2.2).

Bảng 2.5. Thành phần hóa học của thạch cao.

\begin{tabular}{|c|c|c|c|c|c|c|c|c|c|}
\hline $\begin{array}{c}\text { Thành } \\
\text { phần }\end{array}$ & $\mathrm{SiO}_{2}$ & $\mathrm{Al}_{2} \mathrm{O}_{3}$ & $\mathrm{Fe}_{2} \mathrm{O}_{3}$ & $\mathrm{CaO}$ & $\mathrm{MgO}$ & $\mathrm{K}_{2} \mathrm{O}$ & $\mathrm{Na}_{2} \mathrm{O}$ & $\mathrm{SO}_{3}$ & $\mathrm{MKN}$ \\
\hline$\%$ & 0,73 & 0,06 & 0,08 & 31,92 & 0,3 & 0,06 & 0,02 & 43,73 & 21,18 \\
\hline
\end{tabular}

Bảng 2.6. Tính chất cơ lý của thạch cao.

\begin{tabular}{|l|c|}
\hline \multicolumn{1}{|c|}{ Tính chất } & Kết quả \\
\hline 1. Độ ẩm, \% & 29,34 \\
\hline 2. Độ mịn còn lại trên sàng $0,09 \mathrm{~mm}, \%$ & 0 \\
\hline 3. Độ mịn còn lại trên sàng $0,045 \mathrm{~mm}, \%$ & 0,15 \\
\hline
\end{tabular}




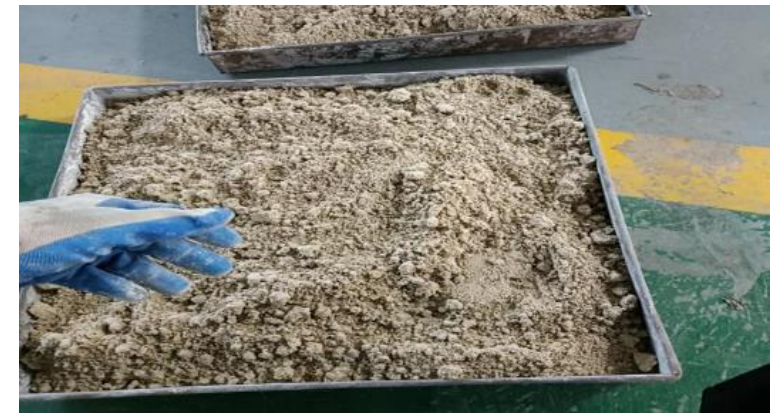

Hình 2.2. Thạch cao FGD Nghi Sơn

\subsubsection{Tro bay nhiệt điện Thăng Long}

Tro bay của nhà máy nhiệt điện Thăng Long được đốt theo công nghệ tầng sôi (CFBC), có độ mịn và độ ẩm được thể hiện trong Bảng 2.7.
Bảng 2.7. Tính chất cơ lý của tro bay.

\begin{tabular}{|l|c|}
\hline \multicolumn{1}{|c|}{ Tính chất } & Kết quả \\
\hline 1. Độ ẩm, \% & 0,5 \\
\hline 2. Độ mịn còn lại trên sàng $0,09 \mathrm{~mm}, \%$ & 2,5 \\
\hline 3. Độ mịn còn lại trên sàng $0,045 \mathrm{~mm}, \%$ & 18,4 \\
\hline
\end{tabular}

\subsubsection{Hạt cốt liệu từ tro bay}

Tro bay được tạo hạt được trình bày tại mục 2.2. Tính chất cơ lý hạt cốt liệu từ tro bay thể hiện trong Bảng 2.8 và Bảng 2.9. Hình dạng hạt được thể hiện trên Hình 2.3.

Hạt cốt liệu từ tro bay kích thước nhỏ hơn $2,5 \mathrm{~mm}$ chiếm số lượng hạt mịn dưới sàng 0,14 mm chiếm 24,98 \%, Trong khi đó hạt cốt liệu từ tro bay kích thước nhỏ hơn $4,5 \mathrm{~mm}$ chiếm số lượng hạt mịn dưới sàng 0,14 mm chiếm nhỏ hơn $2,47 \%$.

Bảng 2.8. Thành phần hạt và các tính chất của cốt liệu từ tro bay có kích thước nhỏ hơn 2,5 mm.

\begin{tabular}{|c|c|c|c|c|c|c|}
\hline Sàng, mm & $\begin{array}{l}\text { Lượng sót riêng, } \\
\qquad a_{i}, \%\end{array}$ & $\begin{array}{l}\text { Lượng sót tích } \\
\text { lũy, } \mathrm{A}_{\mathrm{i}}, \%\end{array}$ & $\begin{array}{c}\text { Mô đun độ } \\
\text { lớn, } \mathrm{M}_{\mathrm{dl}}\end{array}$ & Độ ẩm,\% & $\begin{array}{l}\text { Khối lượng thể tích xốp, } \\
\qquad \rho_{\mathrm{x}}\left(\mathrm{kg} / \mathrm{m}^{3}\right)\end{array}$ & $\begin{array}{l}\text { Khối lượng riêng, } \rho_{\mathrm{a}} \\
\qquad\left(\mathrm{g} / \mathrm{cm}^{3}\right)\end{array}$ \\
\hline 5 & 0 & 0 & \multirow{7}{*}{2,04} & \multirow{7}{*}{10} & \multirow{7}{*}{816} & \multirow{7}{*}{2,29} \\
\hline 2,5 & 0 & 0 & & & & \\
\hline 1,25 & 17,99 & 17,99 & & & & \\
\hline 0,63 & 26,09 & 44,08 & & & & \\
\hline 0,315 & 22,39 & 66,47 & & & & \\
\hline 0,14 & 8,55 & 75,02 & & & & \\
\hline$<0,14$ & 24,98 & 100 & & & & \\
\hline
\end{tabular}

Bảng 2.9. Thành phần hạt và các tính chất của cốt liệu từ tro bay có kích thước nhỏ hơn 4,5 mm.

\begin{tabular}{|c|c|c|c|c|c|c|}
\hline Sàng, mm & $\begin{array}{l}\text { Lượng sót riêng, } \\
\qquad \mathrm{a}_{\mathrm{i}}, \% \\
\end{array}$ & $\begin{array}{l}\text { Lượng sót tích } \\
\text { lũy, } \mathrm{A}_{\mathrm{i}}, \% \\
\end{array}$ & Mô đun độ lớn, $\mathrm{M}_{\mathrm{dl}}$ & Độ ẩm,\% & $\begin{array}{l}\text { Khối lượng thể tích } \\
\text { xốp, } \rho_{x}\left(\mathrm{~kg} / \mathrm{m}^{3}\right)\end{array}$ & $\begin{array}{l}\text { Khối lượng riêng, } \rho_{\mathrm{a}} \\
\left(\mathrm{g} / \mathrm{cm}^{3}\right)\end{array}$ \\
\hline 5 & 0 & 0 & \multirow{7}{*}{3,43} & \multirow{7}{*}{6,06} & \multirow{7}{*}{728} & \multirow{7}{*}{2,11} \\
\hline 2,5 & 32,13 & 32,13 & & & & \\
\hline 1,25 & 21,73 & 53,86 & & & & \\
\hline 0,63 & 17,32 & 71,18 & & & & \\
\hline 0,315 & 16,96 & 88,14 & & & & \\
\hline 0,14 & 9,40 & 97,53 & & & & \\
\hline$<0,14$ & 2,47 & 100 & & & & \\
\hline
\end{tabular}

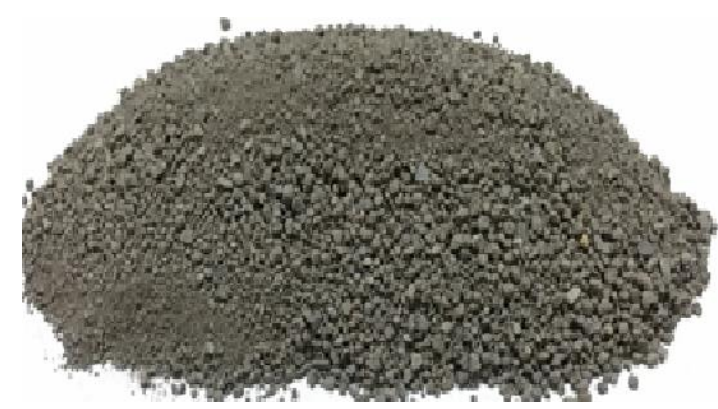

Hình 2.3a. Hạt cốt liệu tro bay kích thước nhỏ hơn 2,5mm.

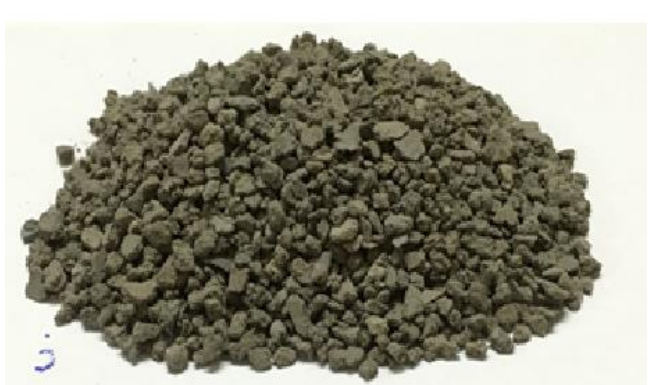

Hình 2.3b. Hạt cốt liệu từ tro bay kích thước nhỏ hơn 4,5mm. 
2.3.5. Cát

Cát vàng có thành phần hạt, độ ẩm và tích chất cơ lý của cát được thể hiện trong Bảng 2.10.

\section{Kết quả nghiên cứu}

Mẫu vữa đối chứng là mẫu sử dụng $100 \%$ cát tự nhiên, cấp phối vữa được khống chế hàm lượng nước để đạt độ chảy từ $11 \mathrm{~cm}$ đến 12 cm, phụ gia hóa học SikaN3000 sử dụng với tỷ lệ 1\% so với khối lượng xi măng, Hạt cốt liệu từ tro bay được thay thế cát với tỷ lệ (10; 20; 30; 40 và 50 ) \% theo khối lượng, Tỷ lệ cấp phối và ký hiệu mẫu được thể hiện trong Bảng 3.1.

Tính chất của vữa sử dụng hạt cốt liệu từ tro bay thay cát có kích thước nhỏ hơn 2,5 mm được thể hiện trong Bảng 3.2.

Từ Bảng 3.2 ta có đồ thị mô tả mối quan hệ giữa hàm lượng hạt cốt liệu từ tro bay thay thế cát tới nhu cầu nước của hỗn hợp vữa, khối lượng thể tích và cường độ nén của vữa được thể hiện trên Hình 3.1; Hình 3.2 và Hình 3.3.

Bảng 2.10. Thành phần hạt và các tính chất của cát tự nhiên.

\begin{tabular}{|c|c|c|c|c|c|c|}
\hline Sàng, mm & $\begin{array}{l}\text { Lượng sót riêng, } \\
\qquad a_{i}, \%\end{array}$ & $\begin{array}{l}\text { Lượng sót tích } \\
\text { lũy, } \mathrm{A}_{\mathrm{i}}, \%\end{array}$ & $\begin{array}{c}\text { Mô đun độ lớn, } \\
\mathrm{M}_{\mathrm{dl}} \\
\end{array}$ & Độ ẩm,\% & $\begin{array}{l}\text { Khối lượng thể tích xốp, } \\
\qquad \rho_{\mathrm{x}}\left(\mathrm{kg} / \mathrm{m}^{3}\right)\end{array}$ & $\begin{array}{c}\text { Khối lượng riêng, } \rho_{\mathrm{a}} \\
\left(\mathrm{g} / \mathrm{cm}^{3}\right)\end{array}$ \\
\hline 5 & 0 & 0 & \multirow{7}{*}{2,75} & \multirow{7}{*}{2,5} & \multirow{7}{*}{1630} & \multirow{7}{*}{2,75} \\
\hline 2,5 & 26,10 & 26,10 & & & & \\
\hline 1,25 & 7,62 & 33,71 & & & & \\
\hline 0,63 & 12,38 & 46,10 & & & & \\
\hline 0,315 & 27,33 & 73,43 & & & & \\
\hline 0,14 & 22,67 & 96,10 & & & & \\
\hline$<0,14$ & 3,90 & 100 & & & & \\
\hline
\end{tabular}

Bảng 3.1. Cấp phối chế tạo mẫu vữa.

\begin{tabular}{|c|c|c|c|c|c|}
\hline \multirow[b]{2}{*}{ Ký hiệu mẫu } & \multicolumn{5}{|c|}{ Tỷ lệ các thành phần, gam } \\
\hline & Xi măng & Cát & $\begin{array}{l}\text { Hạt cốt liệu từ tro bay kích thước } \\
\text { nhỏ hơn } 2,5 \mathrm{~mm}\end{array}$ & $\begin{array}{l}\text { Hạt cốt liệu từ tro bay kích } \\
\text { thước nhỏ hơn } 4,5 \mathrm{~mm}\end{array}$ & Sika N3000 \\
\hline M0 & 450 & 1370 & 0 & 0 & 4,5 \\
\hline TL1 & 450 & 1233 & 137 & 0 & 4,5 \\
\hline TL2 & 450 & 1096 & 274 & 0 & 4,5 \\
\hline TL3 & 450 & 959 & 411 & 0 & 4,5 \\
\hline TL4 & 450 & 822 & 548 & 0 & 4,5 \\
\hline TL5 & 450 & 685 & 685 & 0 & 4,5 \\
\hline TL6 & 450 & 1233 & 0 & 137 & 4,5 \\
\hline TL7 & 450 & 1096 & 0 & 274 & 4,5 \\
\hline TL8 & 450 & 959 & 0 & 411 & 4,5 \\
\hline TL9 & 450 & 822 & 0 & 548 & 4,5 \\
\hline TL10 & 450 & 685 & 0 & 685 & 4,5 \\
\hline
\end{tabular}

Bảng 3.2. Tính chất vữa sử dụng hạt cốt liệu từ tro bay kích thước nhỏ hơn 2,5 mm.

\begin{tabular}{|c|c|c|c|c|c|c|}
\hline \multirow{2}{*}{ Ký hiệu mẫu } & \multirow{2}{*}{ Nước / hỗn hợp } & \multirow{2}{*}{ Độ chảy, cm } & \multirow{2}{*}{$\begin{array}{l}\text { Khối lượng thể tích, } \\
\qquad \mathrm{kg} / \mathrm{m}^{3}\end{array}$} & \multicolumn{3}{|c|}{ Cường độ nén, MPa } \\
\hline & & & & R3 & R7 & $\mathrm{R} 28$ \\
\hline M0 & 0,15 & 11,5 & 2109 & 20,89 & 28 & 31,07 \\
\hline TL1 & 0,16 & 11 & 2055 & 18,55 & 24,97 & 28,39 \\
\hline TL2 & 0,18 & 12,5 & 1993 & 16,37 & 24,25 & 26,34 \\
\hline TL3 & 0,18 & 11,7 & 1955 & 17,47 & 24,08 & 27,54 \\
\hline TL4 & 0,20 & 11 & 1888 & 14,62 & 21,67 & 27,63 \\
\hline TL5 & 0,22 & 11 & 1845 & 13,40 & 20,23 & 24,45 \\
\hline
\end{tabular}




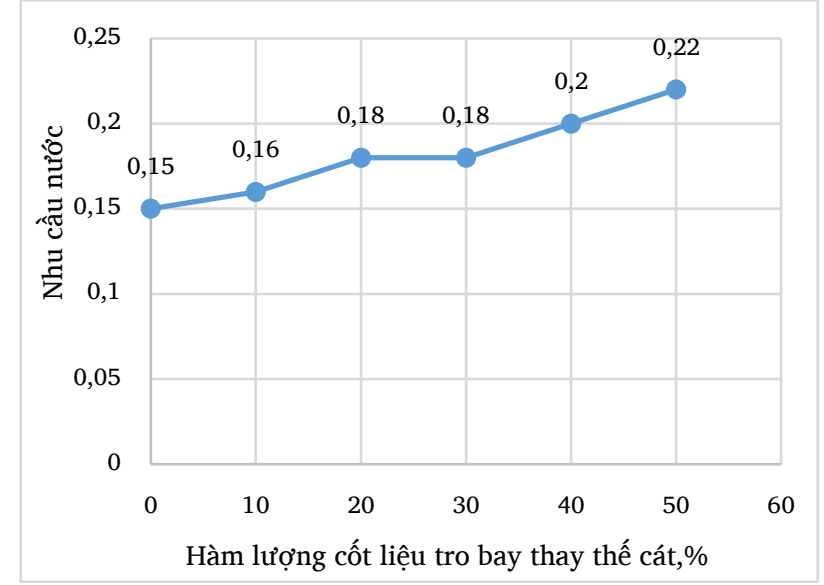

Hình 3.1. Quan hệ giữa hàm lượng hạt cốt liệu từ tro bay với nhu cầu nước của vữa

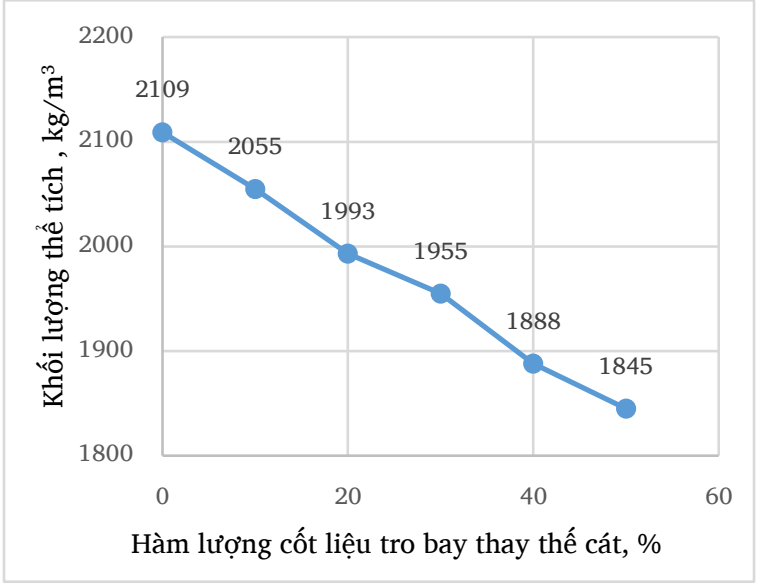

Hình 3.2. Quan hệ giữa hàm lượng hạt cốt liệu từ tro bay với khối lượng thể tích của vữa.

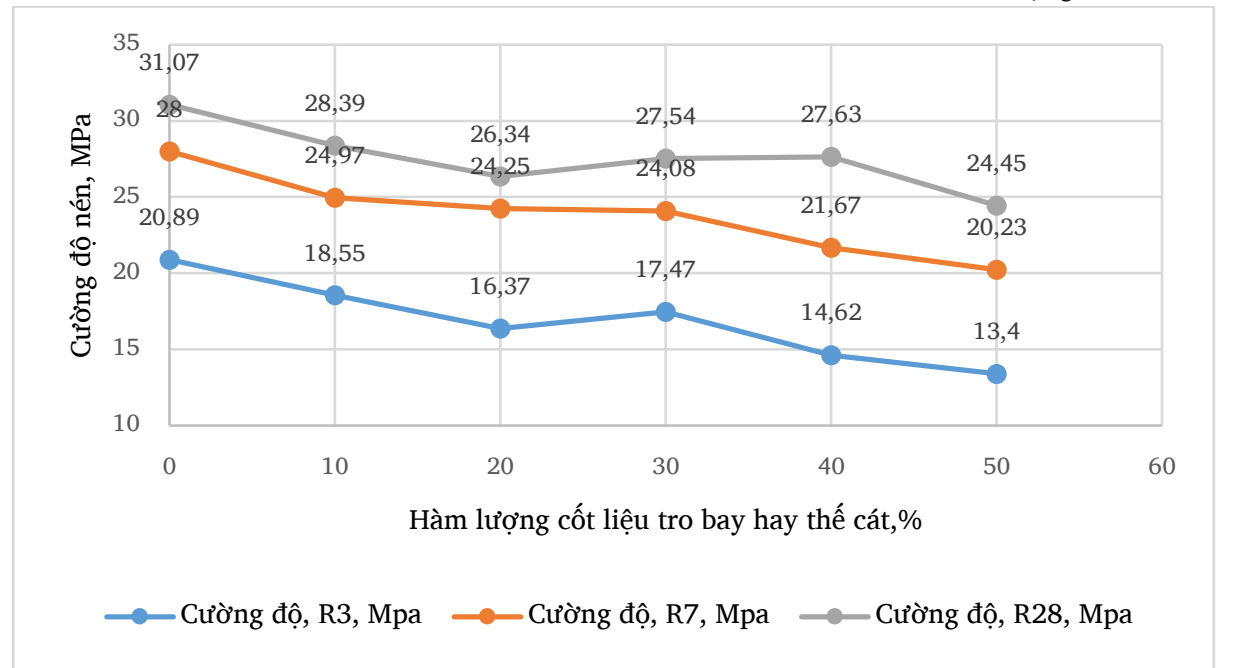

Hình 3.3. Quan hệ giữa hàm lượng cốt liệu từ tro bay với cường độ nén của vữa.

Khi tăng hàm lượng hạt cốt liệu từ tro bay có kích thước hạt nhỏ hơn 2,5 mm thay thế cát trong thành phần của vữa ta có nhận xét sau:

+ Nhu cầu nước của vữa tăng khi tăng hàm lượng cốt liệu tro bay thay thế cát. Vữa có cốt liệu từ tro bay thay thế cát ở tỷ lệ 50 \% , nhu cầu nước tăng $146 \%$ so với mẫu đối chứng, điều này là nguyên nhân giảm cường độ của vữa xi măng. Thêm vào đó nhu cầu nước tăng là do hạt cốt liệu từ tro bay có độ rỗng xốp lớn,

+ Khối lượng thể tích của vữa giảm đáng kể do một phần hạt cốt liệu từ tro bay có khối lượng thể tích thấp hơn so với cát (thể hiện trong Bảng 2.8; 2.9 và 2.10. Mặt khác vữa sử dụng hạt cốt liệu từ tro bay có nhu cầu nước lớn, nước hút vào các rỗ rỗng xốp của các hạt cốt liệu từ tro bay và ngay cả khi bão hòa nước thì khối lượng thể tích hạt cốt liệu từ tro bay vẫn thấp hơn so với trường hợp sử dụng cốt liệu là cát tự nhiên với độ rỗng xốp thấp, hút nước thấp và độ đặc chắc cao.

+ Cường độ nén của vữa giảm khi tăng hạt cốt liệu từ tro bay thay thế cát. Nguyên nhân vữa có chứa lượng lớn hạt cốt liệu từ tro bay mịn có kích thước nhỏ hơn 0,14 mm là 24,98 \% (Bảng 2.8).
Kết quả thử nghiệm tính chất của vữa sử dụng hạt cốt liệu từ tro bay thay cát có kích thước nhỏ hơn 4,5 mm được thể hiện trong Bảng 3.3.

Từ Bảng 3.3 ta có đồ thị mô tả mối quan hệ giữa hàm lượng hạt cốt liệu từ tro bay thay thế cát tới nhu cầu nước của hỗn hợp vữa, khối lượng thể tích và cường độ của vữa được thể hiện trong Hình 3.4; 3.5 và 3.6.

Khi tăng hàm lượng cốt liệu từ tro bay có kích thước hạt nhỏ hơn 4,5 mm thay thế cát trong thành phần của vữa ta có nhận xét sau:

+ Nhu cầu nước của vữa tăng khi hàm lượng cốt liệu từ tro bay thay thế cát $>30 \%$. Tro bay thay thế ở tỷ lệ 50 \%, nhu cầu nước tăng 113 \% so với mẫu đối chứng, nó là nguyên nhân giảm cường độ của vữa xi măng. So sánh kết quả nhu cầu nước của mẫu sử dụng hạt cốt liệu từ tro bay có kích thước nhỏ hơn 2,5mm (Hình 3.1) lớn hơn rất nhiều so với mẫu sử dụng hạt cốt liệu từ tro bay có kích thước nhỏ hơn 4,5 mm (Hình 3.4) ở cùng tỷ lệ thay thế.

+ Khối lượng thể tích của vữa giảm đáng kể khi tăng hàm lượng hạt cốt liệu từ tro bay thay cát, do một phần hạt cốt liệu từ tro bay có khối lượng thể tích nhẹ hơn so với cát (thể hiện trong Bảng 2.8; 2.9 và 
2.10). Mặt khác vữa có sử dụng hạt tro bay nhu cầu nước lớn mẫu đối chứng, nhưng không lớn hơn so với hạt cốt liệu từ tro bay có kích thước $<2,5$ mm (Bảng 3.2 và 3.3 ).

+ Cường độ nén của vữa lớn hơn mẫu đối chứng khi thay thế hạt cốt liệu từ tro bay đến tỷ lệ 30 \% ở tuổi sớm 3 ngày, 7 ngày. Cường độ nén của vữa ở tuổi cường độ 28 ngày hạt cốt liệu từ tro bay thay thế cát 40 \% trong thành phần vẫn nhận được mẫu có cường độ tương đương mẫu đối chứng, Nguyên nhân cường độ tăng ở tuổi dài ngày có thể giải thích là mặc dù tro bay đã được tạo hạt cốt liệu bằng chất kết dính xi măng, nhưng bề mặt các hạt tro bay rất mịn này vẫn có thể xảy ra phản ứng puzolanic với canxi hidroxit - sản phẩm phụ của xi măng khi thủy hóa, làm tăng liên kết giữa xi măng và các hạt tro bay.

Bảng 3.3. Tính chất vữa sử dụng hạt cốt liệu từ tro bay kích thước nhỏ hơn 4,5 mm.

\begin{tabular}{|c|c|c|c|c|c|c|}
\hline \multirow{2}{*}{ Ký hiệu mẫu } & \multirow{2}{*}{ Nước / hỗn hợp } & \multirow{2}{*}{ Độ chảy, cm } & \multirow{2}{*}{$\begin{array}{c}\text { Khối lượng thể } \\
\text { tích, } \mathrm{kg} / \mathrm{m}^{3}\end{array}$} & \multicolumn{3}{|c|}{ Cường độ nén, MPa } \\
\cline { 5 - 8 } & & & 2109 & 20,89 & 28 & R3 \\
\hline M0 & 0,15 & 11,5 & 2077 & 25,73 & 34,35 & 38,25 \\
\hline TL6 & 0,15 & 12 & 1997 & 23,31 & 31,95 & 36,21 \\
\hline TL7 & 0,15 & 11 & 1973 & 21,25 & 26,11 & 33,58 \\
\hline TL8 & 0,16 & 11,6 & 1897 & 18,55 & 23,31 & 30,27 \\
\hline TL9 & 0,16 & 11 & 1858 & 16,25 & 19,74 & 28,02 \\
\hline TL10 & 0,17 & 11,5 & & & & \multicolumn{2}{c|}{ R2 } \\
\hline
\end{tabular}

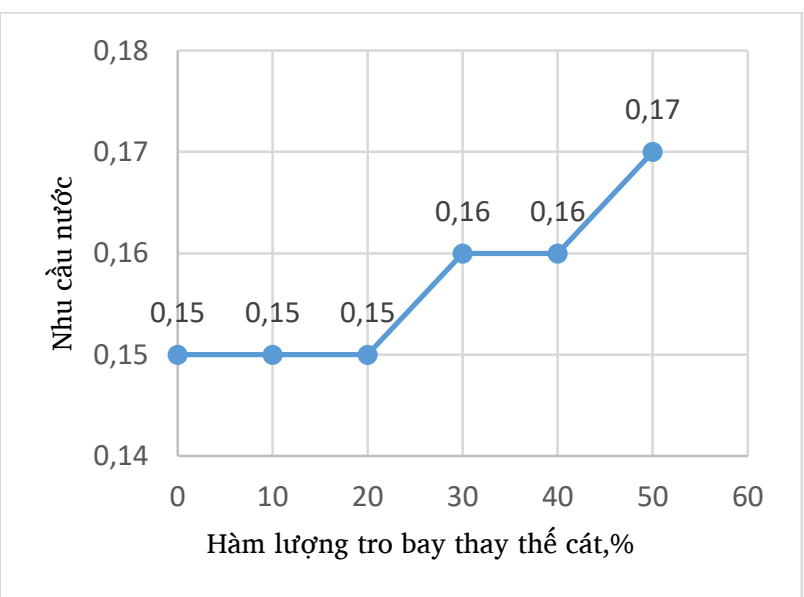

Hình 3.4. Quan hệ giữa hàm lượng cốt liệu từ tro bay với nhu cầu nước của vữa

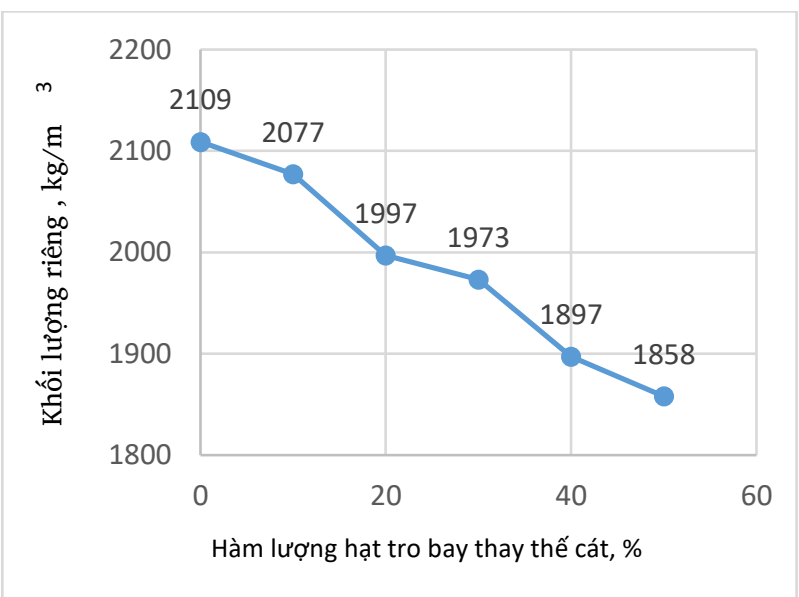

Hình 3.5. Quan hệ giữa hàm lượng cốt liệu từ tro bay với khối lượng thể tích của vữa

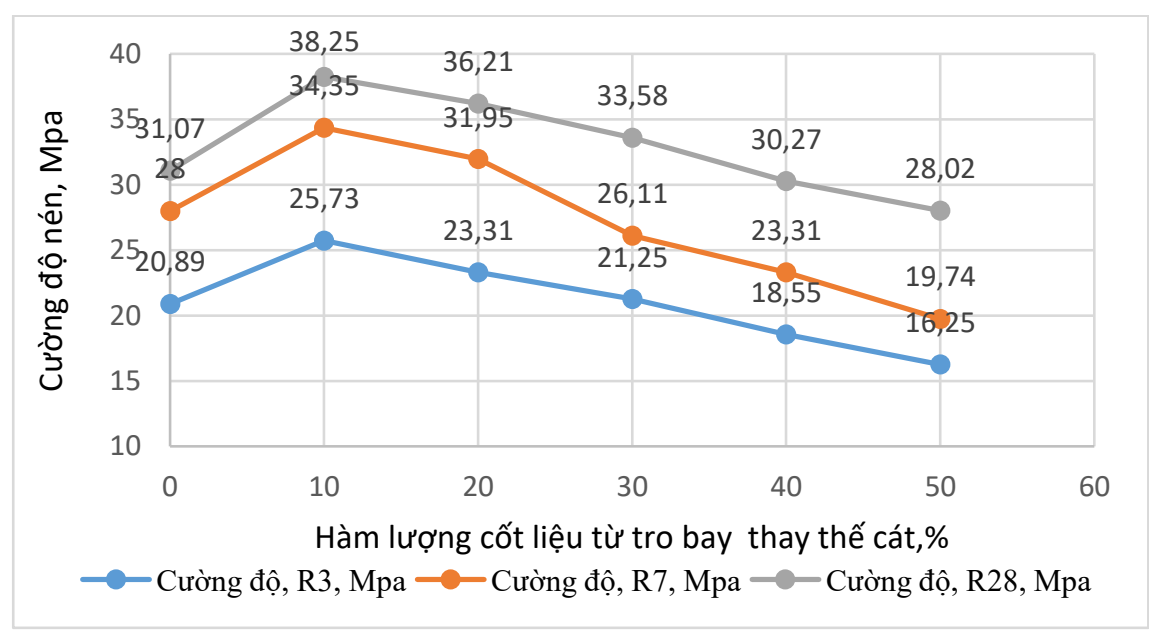

Hình 3.6. Quan hệ giữa hàm lượng cốt liệu từ tro bay với cường độ nén của vữa 


\section{Kết luận}

- Hạt cốt liệu từ tro bay được chế tạo trên thiết bị Vibro Explorer có độ cứng cao, lựa chọn được kích thước hạt theo yêu cầu bằng việc điều chỉnh tỷ lệ nước với hỗn hợp,

- Hạt cốt liệu từ tro bay với kích thước nhỏ hơn 4,5 mm và hàm lượng hạt mịn dưới sàng $0,14 \mathrm{~mm}$ là nhỏ hơn $2,47 \%$ có thể thay thế tới $40 \%$ cát tự nhiên trong thành phần của vữa không làm giảm cường độ của vữa,

- Sử dụng cốt liệu từ tro bay thay cát tự nhiên, nhận được vữa có khối lượng thể tích nhỏ hơn so với mẫu đối chứng. Nếu cốt liệu từ tro bay thay thế cát tới $40 \%$ trong thành phần thì khối lượng thể tích của vữa giảm 10 \% so với mẫu đối chứng,
- Hạt cốt liệu từ tro bay có nhiều lỗ rỗng xốp nhưng với kích thước nhỏ làm tăng khả năng giữ nước nên cần chú ý trước khi sử dụng cốt liệu từ tro bay cần được dưỡng hộ để đạt độ ẩm bão hòa.

\section{Tài liệu tham khảo}

[1]. TS. Lưu Thị Hồng, ThS Trịnh Thị Châm, CN, Lê Duy Văn - Nghiên cứu khả năng tạo hạt thạch cao trên thiết bị Vibro Explorer ; Viện Vật liệu xây dựng Công ty Abe Iron Work, 2021,

[2]. Lê Anh Tài, Huỳnh Phương Nam , Chế tạo cốt liệu nhẹ từ tro bay cho bê tông xi măng, Số 4-2021, trang 26-32, tạp chí Vật liệu \& Xây dựng,

[3]. ASTM C331 / C331M - 17 Standard Specification for Lightweight Aggregates for Concrete Masonry Units

[4]. ASTM C332 - 17 Standard Specification for Lightweight Aggregates for Insulating Concrete 